\title{
Patent foramen ovale and ischemic stroke: more shadows than lights? What the internist should know
}

\author{
Andrea Donti \\ Department of Pediatric Cardiology, S. Orsola-Malpighi Hospital, University of Bologna, Italy
}

\begin{abstract}
The foramen ovale (FO) is an anatomical structure normally present in the fetus, which can persist in up to $25 \%-30 \%$ of the general population after birth. Normally it does not cause any harm but it has been associated with different pathological conditions; the more frequently encountered in clinical practice is an ischemic stroke or transient ischemic attack without any recognizable cause. However, a causal link between the cerebrovascular event and a right to left shunt through the FO is hard to demonstrate in most cases and which is the best treatment for secondary prevention is still a matter of debate with antithrombotic therapy or transcatheter closure of the FO as possible options. No definitive evidence of which is the most effective treatment is actually available so that a careful evaluation of each single patient is mandatory in order to select who will probably benefit the most from the interventional treatment as opposed to who will reasonably do well with medical therapy alone.
\end{abstract}

\section{What is a foramen ovale?}

A foramen ovale (FO) is an anatomical structure allowing communication between the right and the left atrium. Differently from an atrial septal defect which is a hole in the atrial septum, the FO is a tunnel running from right to left between the septum secundum, on the right side, and the septum primum on the left side (Figure 1).

The width and length of the tunnel can be widely different from case to case, depending on the degree

Corrispondence: Andrea Donti, Department of Pediatric Cardiology, S. Orsola-Malpighi Hospital, University of Bologna, via Massarenti 4, 40100 Bologna, Italy.

E-mail: andrea.donti@aosp.bo.it

Key words: Patent foramen ovale; cryptogenic stroke; transcatheter closure.

Acknowledgments: the author would like to thank Dr. Gabriele Egidy Assenza for helping in manuscript editing.

Conflict of interest: the author declares no potential conflict of interest.

Received for publication: 23 November 2015.

Revision received: 16 February 2016.

Accepted for publication: 19 February 2016.

This work is licensed under a Creative Commons Attribution NonCommercial 4.0 License (CC BY-NC 4.0).

CCopyright A. Donti, 2016

Licensee PAGEPress, Italy

Italian Journal of Medicine 2016; 10:175-184

doi:10.4081/itjm.2016.676 of separation and overlapping of the two septa. The septum primum, which is the thinner of the two septa, can be redundant and mobile forming an atrial septal aneurysm and it can harbor one or more fenestrations creating additional communications between the atria. The septum secundum, which is not a real septum but an infolding of the atrial wall filled with fat tissue, can vary in thickness, depending on the amount of fat, up to more than $10 \mathrm{~mm}$ (lipomatous hypertrophy). Therefore, the anatomical features of the FO are consistently variable and additional anatomical structures such as a redundant Eustachian valve or a Chiari network, can further add to the complexity of its anatomy. ${ }^{1}$

The FO is essential for fetal life allowing the oxygenated blood coming from the placenta via the inferior vena cava to cross the atrial septum reaching the left ventricle and ascending aorta to mainly supply the brain; the well-developed Eustachian valve of the fetus plays a crucial role by directing the blood from the inferior vena cava to the entrance of the FO on the right side of the septum.

After birth, respiration lowers the pulmonary vascular resistances increasing the pulmonary blood flow and the blood inflow into the left atrium which, in turn, increases left atrial pressure forcing the septum primum against the septum secundum and functionally closing the FO. With time, the two septa become fused to each other, anatomically sealing the FO. This sealing process does not occur in many people leaving a patent foramen ovale (PFO) in around 25\%-30\% of the general population as shown by autopsy findings and echocardiographic reports. ${ }^{2,3}$

Even if tiny amounts of left to right shunt can occur through a PFO, it mainly acts as a unidirectional valve allowing right to left shunting of blood at the atrial level; any increase of right atrial pressure would 
facilitate right to left shunting but it frequently occurs even with normal atrial pressures.

\section{How to diagnose a patent foramen ovale}

Standard transthoracic ecochardiography (TTE), without saline injection, cannot reliably diagnose a PFO and should not be used for this purpose. Both transcranial Doppler (TCD) and TTE, when performed after injection of agitated saline in a peripheral vein, can make the diagnosis of a right to left shunt with a high sensibility and specificity $(97 \%$ and $93 \%$ for TCD; $91 \%$ and $93 \%$ for TTE); 4,5 performing a Valsalva maneuver is part of both examinations with the aim of transiently and briskly increasing venous return to the right atrium, at releasing of the maneuver, to elicit a right to left shunt in the setting of a PFO.

While TCD can objectively quantify the magnitude of the shunt by counting the high intensity signals recorded at the level of the medial cerebral artery, it cannot identify the site of the shunt, which is not always due to a PFO (e.g., pulmonary fistulas). Even if TTE can add something about the site of shunting, only transesophageal echocardiography (TEE) is able to definitely confirm the PFO as the source of the shunt allowing also to thoroughly delineate its anatomical features which are of special relevance when planning PFO closure. ${ }^{6}$ Moreover, in patients with a history of unexplained ischemic cerebrovascular event, the echocardiographer must always remember to look for any less common possible alternative source of cardioembolism such as atrial mixoma, left atrial appendage thrombosis, left ventricular thrombus or papillary fibroelastomas each of those can coexist even with a large PFO (Figure 2).

\section{When to look for a patent foramen ovale}

A PFO has been associated with many different conditions such as cerebrovascular or systemic ischemia, migraine with aura, transient global amnesia, ortodeoxia-platipnea, obstructive sleep apnea syndrome, and decompression illness in divers.

Nonetheless, given the high prevalence of a PFO in the general population, the effective causal-effect link between the PFO and these conditions is frequently far from being definitely ascertained. Moreover, looking for a PFO seems to be justified only whenever finding it could prompt any interventional or pharmacological treatment at least likely to improve the outcome. This could be the case in patients with cryptogenic cerebrovascular or systemic ischemia, ortodeoxia-platipnea or in professional divers with or without a history of decompression illness. Searching a PFO is frequently asked for in patients who suffer from migraine because case-control studies have shown a higher prevalence of $\mathrm{PFO}$ in migraineurs with aura ( $40 \%$ to $60 \%)$ as compared to the general population $^{7}$ and conversely a higher prevalence of migraine with aura in subjects with a PFO (13\% to $50 \%) .{ }^{8} \mathrm{~A}$ more recent case control study reporting migraine diagnosed by specialists ${ }^{9}$ and a prospective population study about self-reported migraine ${ }^{10}$ failed to confirm this association. Many retrospective studies reported a significant improvement of migraine severity after PFO closure ${ }^{8}$ but the quality of such evidence was really too low to allow any conclusion. As a matter of fact, the MIST trial, a randomized trial comparing PFO closure with medical therapy in patients with severe migraine, failed to demonstrate any substantial benefit of PFO closure with an additional complication rate of $6.8 \%$ in the device group. ${ }^{11}$ Finally, while the presence of white matter lesions on brain magnetic resonance imaging (MRI) is a frequent finding in patients suffering from migraine with aura, no correlation has been found between the white matter lesion load and a right to left shunt at the PFO level. ${ }^{12}$ Other trials about the issue of migraine and PFO are currently underway but, for the time being, there is no evidence to support PFO closure as a mean of treating symptoms or preventing cerebral ischemia.

\section{The occasional diagnosis of a patent foramen ovale}

Not infrequently, an echocardiogram prescribed for different reasons can show an atrial septal aneurysm (prevalence of approximately $2-3 \%$ in the

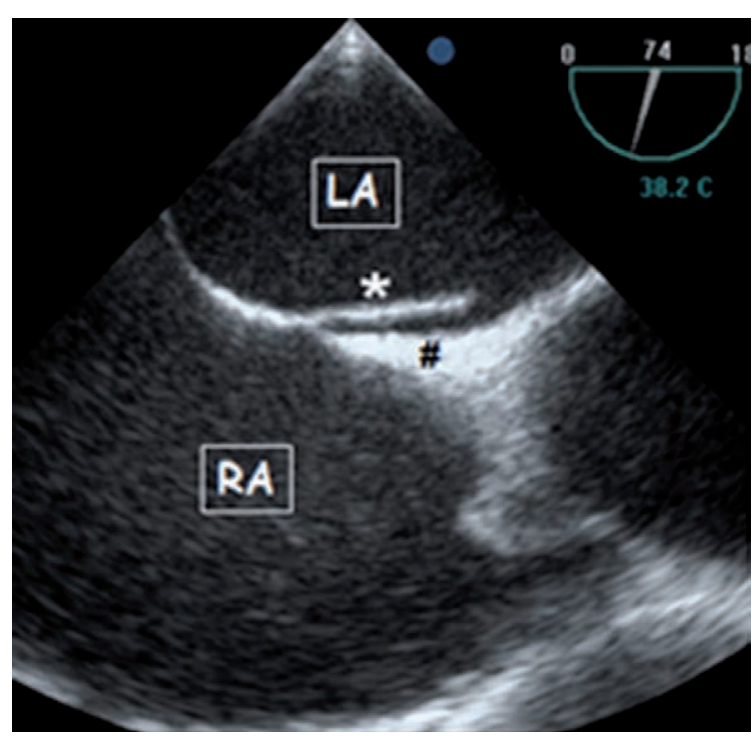

Figure 1. Two-dimensional transesophageal echocardiography long-axis of the patent foramen ovale. LA, left atrium; RA, right atrium; *, septum primum; \#, septum secundum. 
general population $)^{13}$ and or a tiny left to right shunt on color Doppler through a small fenestration of the septum primum. In this setting, a TCD or a TTE with contrast injection is frequently performed to look for a right to left shunt possibly leading to the diagnosis of a PFO. Moreover, a cerebral MRI is not seldom carried out seeking for silent brain lesions and aspirin or even PFO closure are eventually advised, with the aim of preventing paradoxical embolism. This behavior is hardly justified on the basis of our current knowledge. As a matter of fact, two population-based studies have clearly shown that an occasional diagnosis of PFO, alone or associated with an atrial septal aneurysm, do not entail a higher risk of having a first cerebrovascu-
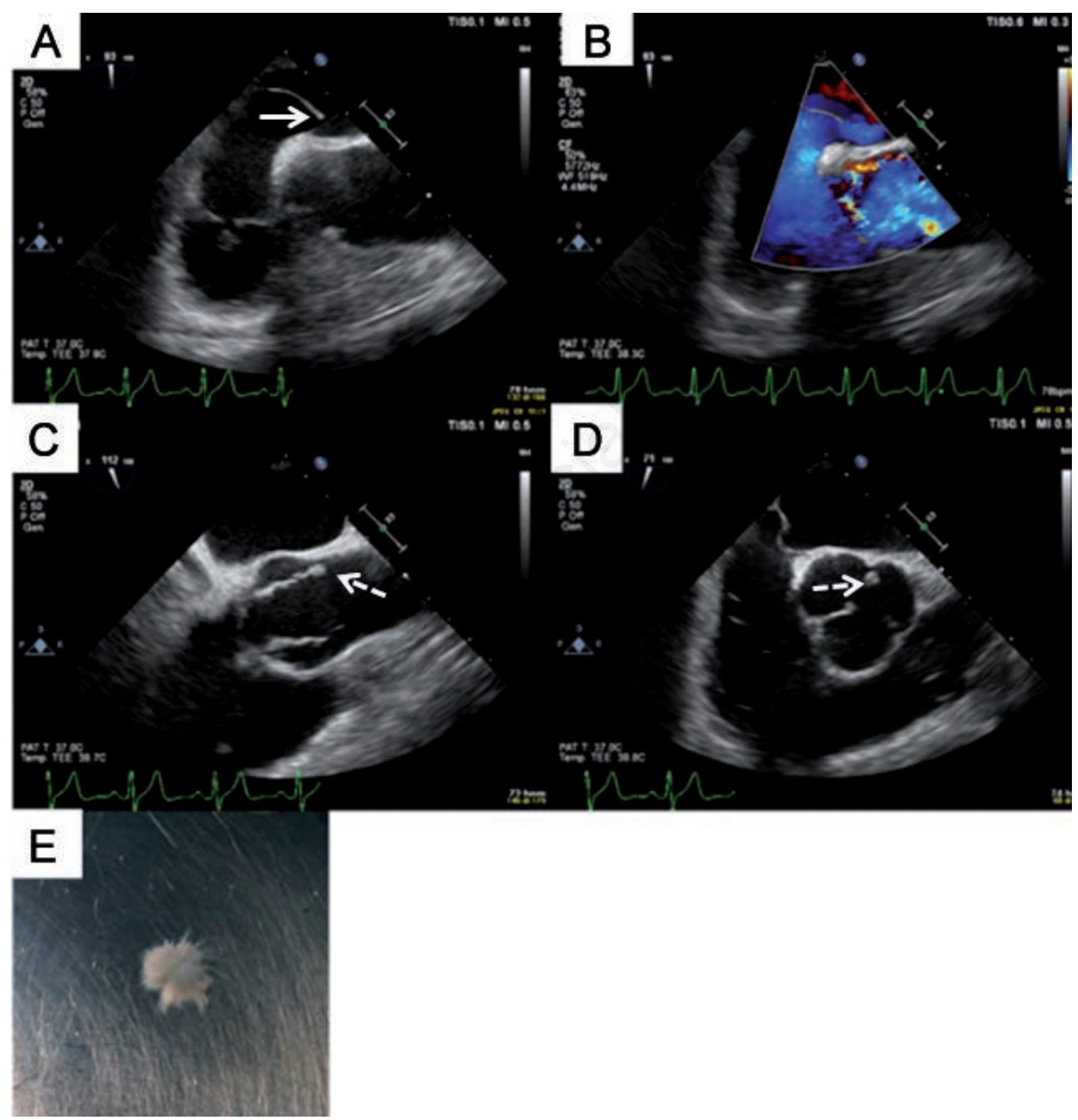

Figure 2. A 54-year-old patient with a hystory of cryptogenic stroke: A) two-dimensional transesophageal echocardiography (2D-TEE) with evidence of a large tunnel of the foramen ovale (FO) (white arrow); B) color-Doppler showing a large right to left shunt through the FO; C) 2D-TEE of the aortic valve in long axis projection showing a small mass adherent to the non-coronary cusp suggestive of a papillary fibroelastoma (dotted arrow); D) the same mass is shown in short axis projection (dotted arrow); E) papillary fibroelastoma of the aortic valve after surgical resection. 
lar event at a mean follow-up of five to seven years as compared to a population without septal anomalies. ${ }^{14,15}$ In the Northern Manhattan Study, enrolling patients over 39 years of age, the hazard ratio (HR) for a first stroke was 1.64 [95\%confidence interval (CI) 0.87-3.09] with a PFO and 1.25 (95\% CI 0.179.29) with PFO and atrial septal aneurysm while in the SPARC study, including patients over 45 years, the HR for a PFO was 1.46 (95\% CI 0.17-2.88). Unexpectedly, an isolated atrial septal aneurysm (without a PFO) was apparently associated with a higher risk of cerebrovascular events but this condition was present only in a small number of patients in both studies and needs further assessment in a larger population.

\section{Patent foramen ovale and cryptogenic cerebrovascular or systemic ischemia}

\section{Are we confident the patent foramen ovale is the culprit?}

Cryptogenic cerebrovascular or systemic ischemia is defined as a cerebrovascular or systemic ischemic episode with no identifiable cause after a complete diagnostic workup. Cryptogenic stroke (CS) is most frequently encountered in clinical practice and it accounts for up to $40 \%$ of ischemic strokes in young patients; a transient ischemic attack (TIA) of undetermined origin is a common finding too. Prevalence of a PFO has been shown to be higher in patients who suffered a CS as compared to patients with a known cause of stroke, mainly under 55 years of age. A metaanalysis of case-control studies showed that patients under 55 years of age with a history of CS were six time more likely to have a PFO as compared to patients with a known cause of stroke ${ }^{16}$ strongly suggesting an association between PFO and CS. The more accepted mechanism accounting for this association is paradoxical embolism defined as thromboembolism originating in the venous system and entering the systemic circulation through the PFO. In situ thrombosis at the PFO or atrial arrhythmias have also been proposed as alternative mechanisms.

The occasional detection of an embolus crossing the PFO in concomitance with a stroke and sometimes with pulmonary thromboembolism, demonstrates the biological plausibility of paradoxical embolization but this is an exception and not the rule. ${ }^{17,18}$ Indeed, a venous source of embolism is only rarely documented in patients with $\mathrm{CS}$ and a PFO; in the recent RESPECT (randomized evaluation of recurrent stroke comparing pfo closure to established current standard of care treatment) trial the prevalence of deep vein thrombosis (DVT) was 3.6\% in the whole study population. ${ }^{19}$ Notably, recent reports suggest that a MRI venogram performed early after a CS can diagnose a peripheral or pelvic venous thrombosis in up to $8 \%$ of the patients with a $\mathrm{PFO}^{20,21}$ also showing congenital anomalies of the pelvic veins possibly predisposing to DVT in an additional $10 \%$ of them..$^{22}$ Combined computed tomography (CT) venography and pulmonary angiography recently yielded similar results with respect to prevalence of DVT, diagnosing a silent pulmonary embolism in $4.4 \%$ of the patients as well. ${ }^{23} \mathrm{In}$ the clinical setting, concomitant occurrence of DVT with or without pulmonary embolism together with systemic embolism in patients with a PFO and a right to left shunt is the only situation allowing a definitive diagnosis of paradoxical embolization.

In daily practice, this chain of events is rarely found and given the high prevalence of a PFO in the general population, an association between PFO and CS does not necessarily imply a causal link explaining the cause of an otherwise CS. This is why the most difficult aspect of the diagnostic workup in each single patient is not to diagnose the PFO but to estimate the probability the PFO would have a causal role and would not be only an incidental finding. The ROPE investigators, by analyzing data coming from 12 databases accounting for more than 3000 patients with CS investigated for a PFO, derived a scoring system (ROPE score) to predict the probability of the stroke being PFO related..$^{24}$ Included in the scoring system were age (top score for the youngest patients and lower for the older ones), absence of hypertension, diabetes and smoke (one point each), no previous TIA or stroke (one point) and type of cerebral lesion on imaging (one point if superficial lesion suggestive of cardioembolism) giving a final score between 0 and 10 . Patients with the higher scores are more likely to have suffered from a PFO related stroke being younger with no or few cardiovascular risk factors and an ischemic cerebral lesion probably due to cardioembolism. Remarkably, patients with the higher probability of having a PFO related stroke also had the lower risk of having a recurrent stroke/TIA at 2-year follow-up (2\% in the higher score stratum versus $20 \%$ in the lower one) suggesting that paradoxical embolism through the PFO is a less common mechanism of stroke as compared to more established causes of cerebral ischemia such as atrial fibrillation or large vessels atherosclerosis. Some anatomical features of the atrial septum such as an atrial septal aneurysm, a large or long PFO tunnel ( $>4 \mathrm{~mm}$ in width and $>10 \mathrm{~mm}$ in length) or the presence of a redundant Eustachian valve ( $>10 \mathrm{~mm}$ in length), have been repeatedly reported to increase the likelihood of paradoxical embolism or the risk of recurrence ${ }^{25-30}$ but conflicting data have been published about this issues ${ }^{31,32}$ so that their positive predictive value seems to be quite low. Nonetheless, more recently, a large right to left shunt as determined by TCD has been shown to be corre- 
lated with a higher ROPE score meaning a plausible PFO related stroke and suggesting an important role for this diagnostic tool in identifying patients more likely to have a pathogenic rather than an incidental PFO. ${ }^{33}$ Moreover echocardiographic features of the atrial septum such as septal hypermobility seem to be associated with a higher risk of recurrence only in patients with a high probability of a PFO related stroke (ROPE score $>6$ ) $\cdot{ }^{34}$ Clinical predictors of paradoxical embolism through a PFO have also been reported in the literature such as a history of DVT/pulmonary embolism, recent prolonged travel, Valsalva maneuver preceding the neurological symptoms or waking up with stroke or TIA. ${ }^{35}$ Finally, some studies also showed a link between inherited thrombophilias such as Factor $\mathrm{V}$ Leiden and mainly the prothrombin G20210A variant and CS with a $\mathrm{PFO}^{36}$ but this has not been confirmed by others. ${ }^{37}$

To summarize for practical purpose, clinical features mainly focusing on age, classical cardiovascular risk factors and history of DVT together with the pattern of cerebral imaging, the modality of occurrence of the neurological symptoms and possibly right to let shunt severity on TCD should help us picking up patients more likely to have a culprit PFO while the anatomical features of the atrial septum seem to be more relevant in predicting the risk of recurrent cerebrovascular events in these patients.

\section{Medical treatment}

Treatment of a stroke, TIA or systemic ischemia likely to be PFO related is still a medical dilemma.

As far as pathophysiology is concerned, systemic embolization in patients with a PFO starts from a platelet-poor thrombus formation either in the venous system, in the tunnel of the PFO or in the left atrium, depending on the involved mechanism (paradoxical embolization, in situ thrombosis or atrial arrhythmias). Accordingly, anticoagulation should be more effective in preventing recurrences as compared to antiplatelet therapy such as in patients with DVT, pulmonary embolism or atrial fibrillation. Nonetheless, a definitive evidence to support this compelling theoretical hypothesis is still not available. A systematic review of observational and randomized data, showed an impressive $50 \%$ reduction in the recurrence risk with warfarin in patients with $\mathrm{CS}$ and $\mathrm{PFO},{ }^{38}$ but a more recent meta-analysis involving more than 2000 patients, did not find a statistically significant difference in the composite outcome of stroke, TIA or death with anticoagulation as compared to antiplatelet therapy. ${ }^{39} \mathrm{De}-$ spite this, the option of using an anticoagulant remains compelling mainly today as the new oral anticoagulants (NOACs) have proved to be very effective and safer than warfarin in patients with venous thromboembolism or atrial fibrillation. To this end, a new neurological entity has been recently accepted, namely the embolic stroke of undetermined source, defined as a non-lacunar infarct without proximal arterial stenosis and major cardioembolic sources ${ }^{40}$ which also include patients with CS and PFO; this new entity has recently been proposed as a possible new target for NOACs which should be tested in randomized trials. ${ }^{41}$

\section{Interventional treatment}

In the last years, transcatheter closure of the PFO has been largely accepted and performed as a means of treating patients with CS and PFO even though no definitive evidence of its superiority over medical therapy is still available. The procedure can be accomplished under general anesthesia with fluoroscopic and TEE monitoring or only with local anesthesia replacing TEE with intracardiac ultrasound. Many different devices are available to close the PFO thus helping fixing different anatomies of the atrial septum. As far as closure effectiveness is concerned, data coming from the recent randomized studies reported complete closure or a small residual shunt on TEE at 6 months follow-up in around $95 \%$ of the cases suggesting that approximately $4 \%$ to $5 \%$ of the patients can still have a significant right to left shunt after device closure. ${ }^{19,42}$ Previous data about accurate TCD follow-up, showed a large residual shunt in $14 \%$ of the cases at a mean of one year post-procedure, irrespective of the type of device but more common in patients who received larger devices $(>33 \mathrm{~mm})$ to close larger PFOs. ${ }^{43}$ Concerning safety, complication rates are generally low but major peri-procedural complications are reported even in the recent years in around $1.5-2.5 \%$ with the most feared ones being cardiac perforation and pericardial tamponade requiring emergency surgery in $0.2-0.4 \%$ of the cases. ${ }^{19,42} \mathrm{~A}$ recent report about more than 7000 procedures of PFO and atrial septal defect closure clearly pointed out that high volume units ( $>38$ procedures/year) had less complications together with a lower length and cost of hospitalization. Device-related complications have also been reported later after PFO closure such as atrial arrhythmias and mainly atrial fibrillation, erosion with pericardial effusion or tamponade and thrombus formation on the device. The incidence of atrial fibrillation as detected by symptoms or standard electrocardiogram (ECG) seems to be around 3\% with the more recent devices $^{19,32}$ as compared to $5.7 \%$ with older ones ${ }^{44}$ even though one report using a 7-day event loop recorder 3 and 6 months after PFO closure detected silent atrial fibrillation of more than $60 \mathrm{~s}$ duration in $15 \%$ of the patients suggesting that silent atrial fibrillation could be a relevant issue. ${ }^{45}$ Further studies are needed to better delineate which is the real incidence of atrial fibrillation after PFO closure and whether it could have a role in stroke recurrences after closure or even in the etiology of the index ischemic event. Real data about late cardiac 
erosion are difficult to obtain but it seems to be extremely rare around $0.01 \%-0.02 \%{ }^{46}$ even though it has been reported up to 10 years after the procedure. ${ }^{47}$ Thrombus formation on the device is difficult to detect unless routine follow-up TEE is performed because it is frequently asymptomatic with a reported incidence around $1 \%-2 \%{ }^{48}$

On the whole, PFO closure today is a substantially safe procedure in experienced centers but close periprocedural monitoring for at least $24 \mathrm{~h}$ and longterm clinical and echocardiographic follow-up are to be warranted.

\section{Interventional treatment versus medical treatment}

Initial observational studies suggested a clear superiority of PFO closure over medical therapy in preventing recurrences in patients with $\mathrm{CS}$ and a PFO. As a matter of fact, two meta-analyses of single-arm or comparative non-randomized studies showed an incidence ratio of recurrent stroke of $0.36-0.76$ events per 100 people years after PFO closure as compared to 2.5-4.4 events per 100 people years with medical therapy, which yielded a statistically significant superiority of the interventional treatment. ${ }^{38,49}$

Unfortunately, these results have not been confirmed by the three randomized clinical trials (RCT) published thus far: CLOSURE-I (evaluation of the starflex septal closure system in patients with a stroke and/or transient ischemic attack due to presumed paradoxical embolism through a patent foramen ovale study), PC (clinical trial comparing percutaneous closure of patent foramen ovale using the Amplatzer ${ }^{T M}$ PFO occluder with medical treatment in patients with cryptogenic embolism trial) and RESPECT.

CLOSURE-I ${ }^{44}$ compared PFO closure using the STARFLEX device with medical therapy in 909 patients under 60 years old with a history of CS or TIA: the incidence of recurrent stroke at 2-year follow-up was $2.9 \%$ in the closure group and $3.1 \%$ in the medical-therapy one $(\mathrm{P}=0.79)$ and the same was true also for recurrent TIA ( $3.1 \%$ vs $4.1 \%$; $\mathrm{P}=0.44)$.

The PC trial ${ }^{42}$ enrolled 414 patients under 60 years old with a history of CS, neuroradiologically verified TIA (symptoms lasting less than $24 \mathrm{~h}$ with an acute ischemic lesion on neuroimaging) or peripheral embolism treated with medical therapy or PFO closure with the Amplatzer ${ }^{\mathrm{TM}}$ device (AGA Medical Corp., Golden Valley, MN, USA). The mean follow-up was longer (4.1 years) but the results were similar with a recurrent stroke rate of $0.5 \%$ with closure and $2.4 \%$ with medical therapy $(\mathrm{P}=0.14)$ and a TIA recurrence rate of respectively $2.5 \%$ and $3.3 \%(\mathrm{P}=0.56)$.

In the CLOSURE-I and the PC trial both the intention to treat and the per-protocol analysis failed to demonstrate a superiority of closure over medical therapy alone.
The RESPECT trial ${ }^{19}$ enrolled 980 patients under 60 years old with a history of ischemic stroke confirmed by cerebral imaging and treated with PFO closure using the Amplatzer ${ }^{\mathrm{TM}}$ device (AGA Medical Corp.) or medical therapy in 1:1 ratio; patients with a TIA or peripheral embolism were excluded. The primary end-point was a composite of recurrent non-fatal ischemic stroke, fatal ischemic stroke or early death after randomization. At a mean follow-up of 2.6 years (range $0-8.1$ years) 25 events occurred, which were all non-fatal strokes; 9 events occurred in the closure group and 16 in the medical-therapy group with no significant difference in an intention to treat analysis [HR with closure 0.49; $\mathrm{P}=0.08]$. Because of a higher drop-out rate in the medical therapy group, as specified in the protocol, the researchers also conducted a per-protocol analysis (HR with closure $0.37 ; \mathrm{P}=0.03$ ) and an as-treated analysis (HR after closure $0.27 ; \mathrm{P}=0.007$ ) both showing a statistically significant superiority of closure over medical therapy. In a subgroup analysis, the protective effect of PFO closure seemed to be more pronounced in patients with an atrial septal aneurysm, a large right to left shunt or a cortical lesion on neuroimaging.

Many meta-analyses have been published about these three RCT giving again conflicting results so that the uncertain conclusions of the RCT themselves have not been clarified by combining their results with quite different meta-analytic approaches.$^{50-55}$ Over all, there is a trend toward a superiority of PFO closure but the quality of the evidence is low and the event reduction with closure seems to be low as well with a high number needed to treat (number of patients to treat in order to avoid one event $>100){ }^{51}$

Moreover, we must aknowledge that all three RCT have substantial limitations deserving some comments: i) the recruitment period was by far longer than predicted, lasting many years, mainly because of difficulties in enrolling patients due to offlabel PFO closure so that patients more likely to have a PFO-related event were possibly treated with closure outside of the trials; ii) difficulties in enrolling patients led to wider inclusion criteria such as in CLOSURE-I which also accepted a TIA as one of the inclusion criteria and primary endpoint with all the well-known biases possibly arising from the clinical diagnosis of a TIA; iii) medical therapy in the medical groups was not standardized but left to the discretion of any participant investigator with approximately $80 \%$ of all the patients treated with aspirin alone. Similarly, type and duration of medical therapy after PFO closure has been different from study to study with patients going on with medical therapy even more than one year after closure (40\% of the patients still on antiplatelets and $5 \%$ on warfarin 4 years after PFO closure in the PC trial); iv) closure I used a device which has been abandoned 
because of a recognized higher risk of device-related complications and is nowadays no more available; $v$ ) the annual recurrence rate was low regardless of the type of treatment, being at most around 1\% per year also in patients on medical therapy alone. As a consequence, the total number of events in the followup was low (25 events in 8-year follow-up in RESPECT) so as to weaken any type of positive or negative conclusion because a wrong attribution of only one or two events to one group or the other would substantially modify the final results.

Other trials are ongoing like the REDUCE (gore septal occluder for patent foramen ovale closure in stroke patients) but they will probably share the same limitations of the previous ones. On the other hand, very recently the long-term results of the RESPECT trial have been presented with a mean follow-up of 5.5 years (J.D. Carrol, unpublished data, 2015); while the intention to treat analysis about any type of recurrent stroke was still negative, looking only to recurrent cryptogenic stroke showed a significant risk reduction with closure [relative risk reduction (RRR) 54\%; $\mathrm{P}=0.042]$ and the same was true looking only at younger patients being less than 60 years old at last follow-up (RRR 52\%; $\mathrm{P}=0.035$ ) suggesting that interventional PFO closure can be superior to medical therapy in carefully selected patients.

The uncertainty coming from the overall evaluation of the randomized and observational studies is also reflected by the recommendations of the international guidelines about this issue.

As a matter of fact, all guidelines underscore that insufficient data are available to make firm recommendations and suggest antiplatelets as the initial treatment for patients with a first CS or TIA and a PFO, provided that no other clinical indication for anticoagulation does exist. Anticoagulants are recommended in case of active venous thrombosis while PFO closure might be considered in patients with a history of DVT provided that no long-term anticoagulation is required or after a recurrent ischemic event while on anticoagulant or antiplatelet therapy (Table 1). ${ }^{55-57}$

Table 1. Recommendations from international guidelines about treatment of patients with ischemic stroke or transient ischemic attack and patent foramen ovale.

\begin{tabular}{ll}
\hline Reference & Recommendations \\
\hline AHA/ASA 20145 & $\begin{array}{l}\text { - For patients with an ischemic stroke or TIA and a PFO who are not undergoing anticoagulation therapy, antiplatelet } \\
\text { therapy is recommended (Class I; Level of Evidence B) }\end{array}$ \\
& $\begin{array}{l}\text { - For patients with an ischemic stroke or TIA and both a PFO and a venous source of embolism, anticoagulation is indi- } \\
\text { cated, depending on stroke characteristics (Class I; Level of Evidence A). When anticoagulation is contraindicated an } \\
\text { inferior vena cava filter is reasonable (Class IIa; Level of Evidence C) }\end{array}$
\end{tabular}

- For patients with a cryptogenic ischemic stroke or TIA and a PFO without Evidence for DVT, available data do not support a benefit for PFO closure (Class III; Level of Evidence A)

- In the setting of PFO and DVT, PFO closure by transcatheter device might be considered, depending on the risk of recurrent DVT (Class IIB; Level of Evidence C)

SPREAD $2015^{56}$ - For patients with a cryptogenic ischemic stroke or TIA and a PFO without DVT or thrombophilia, aspirin $325 \mathrm{mg}$ is indicated

- For patients with a cryptogenic ischemic stroke or TIA and a PFO who are undergoing anticoagulation for other reasons such as DVT or pulmonary embolism, warfarin is indicated

- For patients with a recurrent ischemic stroke or TIA while on antiplatelet or anticoagulant therapy, after a complete multidisciplinary re-evaluation, PFO closure is indicated in accordance with the patient

- Results of the three randomized studies comparing transcatheter PFO closure with medical therapy (CLOSURE-I, PC, RESPECT) do not support PFO transcatheter closure as first line therapy

ACCP $2012^{57} \quad$ - In patients with asymptomatic PFO or atrial septal aneurysm, we suggest against antithrombotic therapy (Grade 2C)

- In patients with cryptogenic stroke and PFO or atrial septal aneurysm, we recommend aspirin (50-100 mg/d) over no aspirin (Grade 1A)

- In patients with cryptogenic stroke and PFO or atrial septal aneurysm, who experience recurrent events despite aspirin therapy, we suggest treatment with VKA therapy (target INR, 2.5; range, 2.0-3.0) and consideration of device closure over aspirin therapy (Grade 2C)

- In patients with cryptogenic stroke and PFO, with evidence of DVT, we recommend VKA therapy for 3 months (target INR, 2.5; range, 2.0-3.0) (Grade 1B) and consideration of device closure over no VKA therapy or aspirin therapy (Grade 2C)

AHA, American Heart Association; ASA, American Stroke Association; TIA, transient ischemic attack; PFO, patent foramen ovale; DVT, deep venous thrombosis; SPREAD, stroke prevention and educational awareness diffusion; CLOSURE-I, evaluation of the starflex septal closure system in patients with a stroke and/or transient ischemic attack due to presumed paradoxical embolism through a patent foramen ovale study; PC, clinical trial comparing percutaneous closure of patent foramen ovale using the Amplatzer ${ }^{\mathrm{TM}}$ (AGA Medical Corp.) PFO occluder with medical treatment in patients with cryptogenic embolism trial; RESPECT, randomized evaluation of recurrent stroke comparing pfo closure to established current standard of care treatment trial; ACCP, American College of Chest Physicians; VKA, vitamin K antagonist; INR, international normalized ratio. 
Cryptogenic stroke or transient ischemic attack in patients with a patent foramen ovale: what to do in clinical practice?

The diagnostic pathway leading to a diagnosis of CS is not a topic of this article but it is mandatory to emphasize that each patient must receive a thorough neurological evaluation aimed at excluding any other possible cause of a cerebrovascular event apart from the PFO. Thereupon, it is noteworthy to remember that searching for silent atrial fibrillation after a CS with prolonged continuous ECG monitoring, has yielded impressive results mainly in patients with a mean age around 60 to 70 years with a detection rate of atrial fibrillation ranging from $12.4 \%$ at 12 months to $16 \%$ at 3 months. ${ }^{58,59}$ Whether similar results could be replicated also in patients less than 60 years of age is still unknown even though a recent report on 98 patients with CS and age less than 50 years, diagnosed atrial fibrillation in $10.2 \%$ of the patients by means of a 3 week Holter monitoring on top of a standard approach, suggesting that looking for atrial fibrillation even in young patients could be rewarding. ${ }^{60}$ Provided that no cause at all has been identified, a screening for right to left shunt should be accomplished preferably by means of TCD or also TTE both with injection of agitated saline in a peripheral vein. A very small shunt (less than 10 high intensity signals on TCD or few isolated bubbles crossing to the left on TTE) should be neglected while a more significant shunt should prompt a TEE examination to confirm the site of the shunt at the PFO level and to delineate its anatomy. Because paradoxical embolism is still the more likely mechanism accounting for a cerebrovascular event in the setting of a PFO, patients should also be screened for a possible source of venous thromboembolism with ultrasonography, MRI venography or CT venography. Blood screening for thrombophilias can be added to the examinations according to medical judgment and clinical history. When a diagnosis of deep venous thrombosis or pulmonary embolism can be done concurrently with the stroke or TIA, paradoxical embolization through the PFO is the very likely cause of the ischemic cerebral event. Otherwise, the likelihood of a PFO related cerebrovascular event should be inferred taking into account all the clinical and instrumental data of each single patient as previously pointed out.

Whether the PFO is judged to be the more likely culprit, antiplatelet therapy should be the first line treatment. Anticoagulation should be probably avoided as chronic treatment, particularly in the youngest patients, provided no other indications does exist apart from the PFO. Transcatheter PFO closure is indicated in patients with a recurrent cerebrovascular event while on well conducted anticoagulation and probably also while on antiplatelet agents, taking into account that superiority of anticoagulation over antiplatelet agents has not been clearly proved up to now.

After a first ever CS or TIA, the evidence supporting PFO closure, for the time being, is undoubtedly weak. Nevertheless, it could be a reasonable option for patients deemed to be very likely to have suffered a PFO related event or to have a higher risk of recurrence on medical therapy alone. Essentially, a young patient (under 60 years) with as few cardiovascular risk factors as possible who suffered a cryptogenic cardiovascular event with evidence of a cerebral superficial ischemic lesion on imaging and with a large right to left shunt, possibly also with an atrial septal aneurysm, is currently the most suitable candidate for PFO closure.

A recent Italian collaborative multidisciplinary position paper summarized the anatomical and clinical risk factors more likely to identify patients who will probably benefit more from an interventional treatment; ${ }^{61}$ according to this paper percutaneous PFO closure is indicated in each patient experiencing a cryptogenic ischemic recurrent event while on anticoagulant or antiplatelet therapy but can also be offered, as an alternative to lifelong medical therapy, to patients with one or more clinical and anatomical risk factors. Anyway, as far as we do not have any definitive evidence about this issue, the final decision should be taken on a case by case base, preferably by a multidisciplinary team (the heart-brain team). Finally, thoroughly informing each patient about potential advantages, disadvantages and uncertainties regarding each kind of therapy is of paramount relevance in the decision-making process.

\section{Conclusions}

A PFO is not a congenital heart disease but rather a normal variant roughly detectable in one quarter of the general population. Occasionally finding a PFO in an asymptomatic individual has no clinical relevance and should not prompt neither any further investigation nor any kind of treatment. The most burdensome situation we have to cope with in clinical practice is cryptogenic stroke or TIA in patients with a right to left shunt through a PFO. Unfortunately, in the vast majority of patients, no definitive causal-effect relationship between the PFO and the cerebrovascular event can be established. Consequently, closing the hole is not necessarily the best solution all the time. As a matter of fact, no definitive evidence is actually available about the best treatment for secondary prevention of recurrences. Transcatheter closure of the PFO is currently a valid option but, waiting for more solid data about its efficacy and safety as compared to medical therapy, it should be offered only to carefully selected patients more likely to have a causal PFO. 


\section{References}

1. Ho SY, McCarthy KP, Rigby ML. Morphological features pertinent to interventional closure of patent oval foramen. J Intervent Cardiol 2003;16:33-8.

2. Hagen PT, Scholz DG, Edwards WD. Incidence and size of patent foramen ovale during the first 10 decades of life: an autopsy study of 965 normal hearts. Mayo Clinic Proc 1984;59:17-20.

3. Meier B, Lock JE. Contemporary management of patent foramen ovale. Circulation 2003;107:5-9.

4. Mojadidi MK, Roberts SC, Winoker JS, et al. Accuracy of transcranial Doppler for the diagnosis of intracardiac right-to-left shunt: a bivariate meta-analysis of prospective studies. JACC Cardiovasc Imaging 2014;7:236-50.

5. Mojadidi MK, Winoker JS, Roberts SC, et al. Two-dimensional echocardiography using second harmonic imaging for the diagnosis of intracardiac right-to-left shunt: a meta-analysis of prospective studies. Int J Cardiovasc Imaging 2014;30:911-23.

6. Mojadidi MK, Bogush N, Caceres JD, et al Diagnostic accuracy of transesophageal echocardiogram for the detection of patent foramen ovale: a meta-analysis. Echocardiography 2014;31:752-8.

7. Schwerzmann M, Nedeltchev K, Lagger F, et al. Prevalence and size of directly detected patent foramen ovale in migraine with aura. Neurology 2005;65:1415-8.

8. Schwedt TJ, Demaerschalk BM, Dodick DW. Patent foramen ovale and migraine: a quantitative systematic review. Cephalalgia Int J Headache 2008;28:531-40.

9. Garg P, Servoss SJ, Wu JC, et al. Lack of association between migraine headache and patent foramen ovale: results of a case-control study. Circulation 2010;121: 1406-12.

10. Rundek T, Elkind MS, Di Tullio MR, et al. Patent foramen ovale and migraine: a cross-sectional study from the Northern Manhattan Study (NOMAS). Circulation 2008;118:1419-24.

11. Dowson A, Mullen MJ, Peatfield R, et al. Migraine Intervention With STARFlex Technology (MIST) trial: a prospective, multicenter, double-blind, sham-controlled trial to evaluate the effectiveness of patent foramen ovale closure with STARFlex septal repair implant to resolve refractory migraine headache. Circulation 2008;117:1397-404.

12. Adami A, Rossato G, Cerini R, et al. Right-to-left shunt does not increase white matter lesion load in migraine with aura patients. Neurology 2008;71:101-7.

13. Agmon Y, Khandheria BK, Meissner I, et al. Frequency of atrial septal aneurysms in patients with cerebral ischemic events. Circulation 1999;99:1942-4.

14. Di Tullio MR, Sacco RL, Sciacca RR, et al. Patent foramen ovale and the risk of ischemic stroke in a multiethnic population. J Am Coll Cardiol 2007;49:797-802.

15. Meissner I, Khandheria BK, Heit JA, et al. Patent foramen ovale: innocent or guilty? Evidence from a prospective population-based study. J Am Coll Cardiol 2006;47:440-5.

16. Overell JR, Bone I, Lees KR. Interatrial septal abnormalities and stroke: a meta-analysis of case-control studies. Neurology 2000;55:1172-9.

17. Hansen A, Kuecherer H. Caught in the act: entrapped embolus through a patent foramen ovale. Eur J Echocardiogr 2008;9:692-3.
18. Bugra Z, Hunerel D, Tayyareci Y, et al. Echocardiographic diagnosis of a giant thrombus passing through a patent foramen ovale from right atrium to the left atrium. J Thromb Thrombolysis 2008;25:297-9.

19. Carroll JD, Saver JL, Thaler DE, et al. Closure of patent foramen ovale versus medical therapy after cryptogenic stroke. N Engl J Med 2013;368:1092-100.

20. Cramer SC, Rordorf G, Maki JH, et al. Increased pelvic vein thrombi in cryptogenic stroke: results of the Paradoxical Emboli from Large Veins in Ischemic Stroke (PELVIS) study. Stroke 2004;35:46-50.

21. Liberman AL, Daruwalla VJ, Collins JD, et al. Diagnostic yield of pelvic magnetic resonance venography in patients with cryptogenic stroke and patent foramen ovale. Stroke 2014;45:2324-9.

22. Osgood M, Budman E, Carandang R, et al. Prevalence of pelvic vein pathology in patients with cryptogenic stroke and patent foramen ovale undergoing MRV pelvis. Cerebrovasc Dis 2015;39:216-23.

23. Lapergue B, Decroix JP, Evrard S, et al. Diagnostic yield of venous thrombosis and pulmonary embolism by combined CT venography and pulmonary angiography in patients with cryptogenic stroke and patent foramen ovale. Eur Neurol 2015;74:69-72.

24. Kent DM, Ruthazer R, Weimar C, et al. An index to identify stroke-related vs incidental patent foramen ovale in cryptogenic stroke. Neurology 2013;81:619-25.

25. Mas JL, Arquizan C, Lamy C, et al. Recurrent cerebrovascular events associated with patent foramen ovale, atrial septal aneurysm, or both. N Engl J Med 2001;345: 1740-6.

26. Homma S, Di Tullio MR, Sacco RL, et al. Characteristics of patent foramen ovale associated with cryptogenic stroke. A biplane transesophageal echocardiographic study. Stroke 1994;25:582-6.

27. De Castro S, Cartoni D, Fiorelli M, et al. Morphological and functional characteristics of patent foramen ovale and their embolic implications. Stroke 2000;31:2407-13.

28. Schuchlenz HW, Weihs W, Horner S, Quehenberger F. The association between the diameter of a patent foramen ovale and the risk of embolic cerebrovascular events. Am J Med 2000;109:456-62.

29. Giardini A, Donti A, Formigari R, et al. Spontaneous large right-to-left shunt and migraine headache with aura are risk factors for recurrent stroke in patients with a patent foramen ovale. Int J Cardiol 2007;120:357-62.

30. Vale TA, Newton JD, Orchard E, et al. Prominence of the Eustachian valve in paradoxical embolism. Eur J Echocardiogr 2011;12:33-6.

31. Serena J, Marti-Fabregas J, Santamarina E, et al. Recurrent stroke and massive right-to-left shunt: results from the prospective Spanish multicenter (CODICIA) study. Stroke 2008;39:3131-6.

32. Homma S, Sacco RL, Di Tullio MR, et al. PFOiCSS. Effect of medical treatment in stroke patients with patent foramen ovale: patent foramen ovale in cryptogenic stroke study. Circulation 2002;105:2625-31.

33. Wessler BS, Kent DM, Thaler DE, et al. The RoPE score and right-to-left shunt severity by transcranial Doppler in the CODICIA study. Cerebrovasc Dis 2015;40:52-8.

34. Thaler DE, Ruthazer R, Weimar C, et al. Recurrent stroke predictors differ in medically treated patients with pathogenic vs. other PFOs. Neurology 2014;83:221-6.

35. Ozdemir AO, Tamayo A, Munoz C, et al. Cryptogenic 
stroke and patent foramen ovale: clinical clues to paradoxical embolism. J Neurol Sci 2008;275:121-7.

36. Pezzini A, Del Zotto E, Magoni M, et al. Inherited thrombophilic disorders in young adults with ischemic stroke and patent foramen ovale. Stroke 2003;34:28-33.

37. Favaretto E, Sartori M, Conti E, et al. G1691A factor V and G20210A FII mutations, acute ischemic stroke of unknown cause, and patent foramen ovale. Thrombosis Res 2012;130:720-4.

38. Kitsios GD, Dahabreh IJ, Abu Dabrh AM, et al. Patent foramen ovale closure and medical treatments for secondary stroke prevention: a systematic review of observational and randomized evidence. Stroke 2012;43: 422-31.

39. Kent DM, Dahabreh IJ, Ruthazer R, et al. Anticoagulant vs. antiplatelet therapy in patients with cryptogenic stroke and patent foramen ovale: an individual participant data meta-analysis. Eur Heart J 2015;36:2381-9.

40. Hart RG, Diener HC, Connolly SJ. Embolic strokes of undetermined source: support for a new clinical construct-authors' reply. Lancet Neurol 2014;13:967.

41. Yaghi S, Kamel H, Elkind MS. Potential new uses of non-vitamin $\mathrm{K}$ antagonist oral anticoagulants to treat and prevent stroke. Neurology 2015;85:1078-84.

42. Meier B, Kalesan B, Mattle HP, et al. Percutaneous closure of patent foramen ovale in cryptogenic embolism. N Engl J Med 2013;368:1083-91.

43. Harms V, Reisman M, Fuller CJ, et al. Outcomes after transcatheter closure of patent foramen ovale in patients with paradoxical embolism. Am J Cardiol 2007;99: 1312-5.

44. Furlan AJ, Reisman M, Massaro J, et al. Closure or medical therapy for cryptogenic stroke with patent foramen ovale. N Engl J Med 2012;366:991-9.

45. Burow A, Schwerzmann M, Wallmann D, et al. Atrial fibrillation following device closure of patent foramen ovale. Cardiology 2008;111:47-50.

46. Amin Z, Hijazi ZM, Bass JL, et al. PFO closure complications from the AGA registry. Catheter Cardiovasc Intervent 2008;72(1):74-9.

47. Vitanova K, Cleuziou J, Vogt M, et al. Delayed perforation of a transcatheter patent foramen ovale occluder 10 years after implantation. Annals Thorac Surg 2015;99: 700-1.

48. Abaci A, Unlu S, Alsancak Y, et al. Short and long term complications of device closure of atrial septal defect and patent foramen ovale: meta-analysis of 28,142 patients from 203 studies. Catheter Cardiovasc Intervent 2013;82:1123-38.

49. Agarwal S, Bajaj NS, Kumbhani DJ, et al. Meta-analysis of transcatheter closure versus medical therapy for patent foramen ovale in prevention of recurrent neurological events after presumed paradoxical embolism. JACC Cardiovasc Intervent 2012;5:777-89.

50. Stortecky S, da Costa BR, Mattle HP, et al. Percutaneous closure of patent foramen ovale in patients with cryptogenic embolism: a network meta-analysis. Eur Heart J 2015;36:120-8

51. Kitsios GD, Thaler DE, Kent DM. Potentially large yet uncertain benefits: a meta-analysis of patent foramen ovale closure trials. Stroke 2013;44:2640-3.

52. Pickett CA, Villines TC, Ferguson MA, Hulten EA. Percutaneous closure versus medical therapy alone for cryptogenic stroke patients with a patent foramen ovale: meta-analysis of randomized controlled trials. Texas Heart Institute J 2014;41:357-67.

53. Benkhadra K, Wang Z, Murad MH. Network metaanalysis: introduction and an example that compares devices for PFO closure. Eur Heart J 2015;36:80-2.

54. Li J, Liu J, Liu M, et al. Closure versus medical therapy for preventing recurrent stroke in patients with patent foramen ovale and a history of cryptogenic stroke or transient ischemic attack. Cochrane Database Syst Rev 2015;9:CD009938.

55. Kernan WN, Ovbiagele B, Black HR, et al. Guidelines for the prevention of stroke in patients with stroke and transient ischemic attack: a guideline for healthcare professionals from the American Heart Association/American Stroke Association. Stroke 2014;45:2160-236.

56. Italian Stroke Organization (ISO); Stroke Prevention and Educational Awareness Diffusion (SPREAD). Linee Guida ISO-SPREAD; 2015. Available from: http:// www.iso-spread.it/index.php

57. Guyatt GH, Akl EA,Crowther M, et al. Antithrombotic therapy and prevention of thrombosis, 9th ed. American College of Chest Physician Evidence-Based Clinical Practice Guidelines. Chest 2012;141:7S-47S.

58. Sanna T, Diener HC, Passman RS, et al. Cryptogenic stroke and underlying atrial fibrillation. N Engl J Med 2014;370:2478-86.

59. Gladstone DJ, Spring M, Dorian P, et al. Atrial fibrillation in patients with cryptogenic stroke. N Engl J Med 2014;370:2467-77.

60. Sanak D, Hutyra M, Kral M, et al. Atrial fibrillation in young ischemic stroke patients: an underestimated cause? Eur Neurol 2015;73:158-63.

61. Pristipino C, Anzola GP, Ballerini L, et al. Management of patients with patent foramen ovale and cryptogenic stroke: a collaborative, multidisciplinary, position paper. Catheter Cardiovasc Intervent 2013;82:E38-51. 